$\begin{array}{lllllllllllllllllllll}A & R & T & I & C & U & L & O & D & E\end{array}$ $\begin{array}{lllllllll}R & E & F & L & E & X & I & O & N\end{array}$

\title{
LA CONSTRUCCIÓN DE LA ESCRITURA A TRAVÉS DEL CUENTO1
}

\author{
THE CONSTRUCTION OF WRITING THROUGH THE STORY
}

\author{
Por: Yazmín Molano Castañeda*
}

*Licenciada en Educación Básica con Énfasis en Humanidades y Lengua Castellana de la Universidad Distrital Francisco José de Caldas. Actualmente candidata para Magíster en Pedagogía de la Lengua Materna de la Universidad Distrital Francisco José de Caldas. E-mail: molanoyaz@gmail. com

1 Este artículo surge desde la investigación realizada en la Maestría en Pedagogía de la Lengua Materna de la Universidad Distrital Francisco José de Caldas, titulada: "El juego en la creación de cuentos en grado cuarto" la cual inició en el año 2015 y aún se encuentra en curso. Esta investigación se instala en la línea: pedagogía de las actividades discursivas de la maestría.
Recibido: 1 de marzo de 2017 - Aprobado: 5 de julio de 2017

\section{RESUMEN}

En la escuela se ha percibido y vivido la escritura como una transcripción, lo que ha hecho que los estudiantes se alejen cada vez más del significado que conlleva este importante proceso. Asimismo, la escritura requiere dedicación e interés en donde es necesario reestructurar la mente y salir de ciertos paradigmas en los cuales se ha encasillado como ingenua. Este artículo busca reflexionar sobre nuevas formas de enseñanza de la escritura a partir del cuento literario, pues es gracias a él que es posible escapar de una escritura sin mayor importancia para que esta se convierta en un proceso escritural real, desplegando el poder que tiene la palabra y la trascendencia que tiene la creación de una historia. Esta reflexión hace parte del proyecto de investigación titulado El juego en la creación de cuentos en grado cuarto, el cual se realiza con los estudiantes de grado cuarto de un colegio de la ciudad de Bogotá y que nació en el marco de la Maestría en Pedagogía de la Lengua Materna de la Universidad Distrital.

Palabras clave: escritura, escritura proceso, cuento literario y taller pedagógico.

\begin{abstract}
At school, writing has been perceived and lived as a transcript, which has led students to move away from the meaning of this important process. Likewise, writing requires dedication and interest where it is necessary to restructure the mind and leave certain paradigms in which it has been labeled as naive. This article seeks to reflect on new ways of teaching writing from the literary story, because it is thanks to it that it is possible to escape from a writing without major importance to become a real scriptural process, deploying the power that the word has and transcendence that the creation of a story has. This reflection is part of the research project entitled The game in the creation of stories in fourth grade, which is done with the fourth grade students of a school in the city of Bogota and was born within the framework of the Master in Pedagogy of the Mother Tongue at the District University.
\end{abstract}

Keywords: writing, writing process, literary story and teaching workshop.

\section{Introducción}

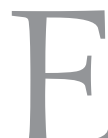
n la actualidad la escuela se encuentra regida bajo la concepción de que la escritura se debe centrar en la decodificación y en la producción en masa de gran cantidad de textos sin importar cómo fueron construidos. 
En estos textos es fácil encontrar que se evalúa un producto inmediato bajo criterios que buscan complacer al docente, más no se evidencia una construcción de sentido. Este panorama permite visibilizar que escribir se convierte en un ejercicio momentáneo que no responde a unas consignas claras y que poco justifican la aprehensión de los estudiantes frente a un tipo de texto específico.

Lo anterior conlleva a pensar que se escribe sin dar la importancia suficiente a este acto, es decir, se debe ir más allá de las reglas gramaticales y de la normatividad para que se pueda encontrar el significado de lo que es escribir, para que el estudiante pueda dimensionar que esta actividad discursiva tiene trascendencia como lo señala Céspedes (2014) quien hace alusión a Ferreiro al señalar que tanto la lectura como la escritura deben estar relacionadas con su cotidianidad. Por esta razón, es importante resaltar que en ocasiones resulta lamentable percibir que se le otorga mayor relevancia a aspectos formales de la lengua, como el uso del espacio, la letra y la ortografía.

Pero es fundamental abrir la brecha a nuevos conocimientos; si la escritura se construye haciendo por qué no abarcar otros elementos que hacen que el estudiante tenga que ponerse en escena, en donde requiere pensar y reflexionar para poder escribir algo que contribuya en su entorno, que tenga en cuenta que esta actividad discursiva de la lengua cumple una función social y no es un instrumento estático, sino que es dinámica porque permite reflexionar, defender ideas y compartir infinidad de opiniones respecto al contexto que nos rodea.

La escuela y los docentes deben hacer un acercamiento a la escritura, en donde los niños puedan identificar que esta es un proceso, entendiendo este término desde Tolchinsky (1993) como las fases, la generación de ideas y la planificación para hacer un texto, lo que conlleva a la realización de un plan que permita organizar su pensamiento y en el que el estudiante pueda comprender para qué escribe y cómo lo escribe.

Pero lo que se evidencia es que en algunas aulas la escritura se asocia a actividades que causan cansancio, creando en los estudiantes el imaginario en donde esta es una obligación o peor aún relacionándola con aspectos negativos. Un ejemplo claro es cuando los docentes la usan como un medio de castigo (para hacer planas) porque se desdibuja totalmente su propósito, lo que conlleva a que pierda la importancia que tiene, que la palabra se 
desvanezca y se compare con el hastío, con el regaño, con la imposición y se aleje del goce, de la aventura y hasta de la creatividad.

A causa de esto, es que se debe concebir la escritura desde una perspectiva diferente a la actual, como lo menciona Bernardin (2014) se requiere que haya una apropiación; si el estudiante no tiene la ruta clara de escritura es prácticamente imposible que pueda visualizar que esta actividad discursiva tiene un fin, que no se hace partiendo de la nada, sino que por el contrario es necesario la elaboración y utilización de estrategias que harán que su escrito sea exitoso. Pero esto se logra cuando el maestro le muestra una ruta diferente como se hace en el proyecto "El juego en la creación de cuentos en grado cuarto", en donde prima el acercamiento del estudiante con ese mundo de palabras que va creando e hilando, porque se hace notorio el interés por la escritura de cuentos.

De igual forma autores como Scardamalia y Bereiter (1992) consideran que: "además del mejoramiento del estilo, el alumno muestra un adelanto estructural importante" (p.54) lo que indica que la preocupación no se da por aspectos como el trazo, la caligrafía y la ortografía, sino que pretende que el sujeto pueda comprender que un texto tiene una situación inicial, una complicación o situación desencadenante y una solución como lo postuló el profesor Jean Michel Adam (1992). Así pues este artículo muestra la escritura como un proceso y no como un producto en el que se toma el tiempo necesario para planificar y revisar, que son a su vez pasos que no se tienen en cuenta al momento de generar los diversos escritos.

\section{La escritura como proceso y no como producto}

7 l preguntar a algunos maestros sobre cómo conciben la escritura en sus clases si como un producto o un proceso, automáticamente

contestan que esta debería ser considerada como un proceso; no obstante, sus prácticas de aula son totalmente diferentes porque se tiene la presión de la academia por dar una nota al estudiante y se juega al error y acierto. Es decir, en el proceso el estudiante tuvo que esforzarse mucho pero logró avances significativos, sin embargo al presentar su escrito se evidenciaron varias fallas y lo que se hace desde la escuela es otorgar una nota baja porque no llegó a lo que se esperaba, es decir su proceso no es formativo sino que se recurre a los métodos tradicionales en los que la escritura del estudiante debe mostrar resultados efectivos pero en ningún momento se le ha hecho consciente de lo que implica este acto. 
El ideal es que tanto estudiantes como maestros pensemos en una escritura proceso, en la que el estudiante aprenda a hacer un plan de escritura, que tenga en cuenta que al igual que irse de viaje requiere con precisión saber la ruta o camino que debe seguir pero lo que se hace en la realidad es arrojar un serie de elementos y decirle al estudiante "escriba" como si fuera arrojado a un mundo desconocido en donde no cuenta con las herramientas necesarias, donde es probable que en el primer acercamiento sea derrotado. Entonces la escritura es valorada de forma cuantitativa perdiendo su sentido comunicativo.

Por lo tanto, como lo afirma Ferreiro (1998), es necesario no construir una escritura sino reconstruirla, quitar esos imaginarios que la escuela se ha encargado de reproducir año tras año de escolaridad porque se ha pensado que quien escribe con buena ortografía y haciendo uso de las reglas gramaticales es superior en este ámbito, desconociendo elementos estructurales que en realidad si conllevan a la reflexión. Ferreiro (1998) es puntual al decir que "tienen que reconstruirla para poder apropiársela" (p.159) es decir, que resulta complejo percibir la escritura como proceso si se continúan las mismas prácticas devastadoras que conducen a que la escritura es similar al dictado y que fuera de este no hay nada más, desconociendo la escritura creativa a la que apunta Gianni Rodari (1983), en donde se parte de elementos relativamente sencillos para generar historias sorprendentes.

Por lo tanto, es necesario tener visión y proyección al momento de enseñar a escribir y fortalecer esa escritura a través de proyectos, secuencias didácticas o talleres pedagógicos que tengan significado, que se trace un recorrido en el que se le brinde al estudiante una serie de elementos y pueda ir avanzando en su escritura, se requiere del acompañamiento del maestro para hacer posible esa escritura proceso y que el estudiante se familiarice con la reescritura, que más allá de elementos puramente estilísticos, se deben enseñar elementos que apunten a la estructura, a la utilización de un vocabulario más amplio, etc.

Como lo señala Cassany (1995), es necesario tener en cuenta la estructura del texto que se piensa realizar, porque "muchos tipos de texto tienen una estructura estandarizada. Así, una carta tiene cabecera, introducción, cuerpo y conclusión (...) estas convenciones facilitan notablemente el trabajo del escritor" (Cassany, 1995: 76), en el caso del cuento reconocer cómo se inicia una historia y cómo culmina es fundamental porque de lo contrario la escritura no tiene un propósito claro. 


\section{El cuento infantil}

n relación con lo anterior, se ha iniciado con una propuesta que busca tener grandes resultados porque apunta a la escritura proceso a través del cuento, pero no haciendo de este un instrumento de trabajo, sino que se pretende llegar a la construcción de significado siguiendo las pautas dadas. Se busca alejar la concepción errónea y poco inadecuada que se tiene de escritura para que se pueda reconocer al sujeto como un ser activo en el proceso, que comete errores pero que logra perfeccionar su escritura gracias a la reescritura. En donde el maestro le señala sus debilidades pero también sus fortalezas, que lo potencia desde lo positivo para que pueda avanzar y no solo se queda en resaltar las fallas.

Esto es lo que sucede en el proyecto "El juego en la creación de cuentos en grado cuarto" en el que se evidencian resultados positivos al momento de permitirle al estudiante que continúe su escrito así no tenga buena ortografía o la letra solicitada pero en el que se logra que fluya su imaginación y que no sienta limitantes que causan un estancamiento de la escritura, de esta manera el proyecto ha impactado a los niños en tanto es visto como una forma diferente de escribir, el cual se encarga primero de atrapar al estudiante

y a medida que logra involucrarse se le puedan brindar elementos que hacen que sus escritos se puedan ir perfeccionando.

Se debe agregar que como afirma Nigro (2006) "La escritura, como la lectura, es un proceso, es decir, que se va dando paso a paso y que requiere de un tiempo de maduración para que se alcancen los objetivos comunicacionales del que escribe, para que el texto goce de claridad y de eficacia" (p.122), esto apunta a que la escritura no es algo acabado simultáneamente, sino que requiere varias relecturas y estar en constante reflexión para asegurarse que lo que está plasmado efectivamente cumpla con las expectativas. La escritura no es una actividad discursiva que se haga con gran facilidad, por el contrario escribir de forma adecuada lleva tiempo y mucho más lograr comprender por qué la escritura debe hacerse de forma progresiva y no de forma precipitada.

Por lo tanto, el cuento literario será el foco sobre el que se centrará la investigación, esto con el fin de hacer la invitación a una escritura más amena y que mientras se crean los propios cuentos, los estudiantes observarán la funcionalidad de distintos elementos como el uso de los conectores, entenderán la estructura y no solo memorizarán que tiene un inicio, nudo y desenlace, 
sino que el estudiante logrará la aprehensión y, por tanto, su cuento ya no será realizado desde lo innato sino poniendo en praxis lo que se le ha enseñado, es como lo señala Ander-Egg es un aprender haciendo.

Lo que se busca es que estos elementos que parecen ser tradicionales el estudiante pueda comprender su relevancia más allá de una identificación que no conlleva a un análisis y reflexión debido a que esto sucedía en el proyecto "El juego en la creación de cuentos en grado cuarto" en el que los niños identificaban un inicio, nudo y final de una historia, pero lo hacían más de forma visual que teniendo en cuenta el cuento, al igual que la escritura de un título, siempre empezaban con la escritura de este sin antes hacer su cuento y dejar para el final este para recopilar todo lo que habían escrito.

Para que esta experiencia resulte benéfica, se parte desde la Gramática de la fantasía de Gianni Rodari (1983), quien hace una invitación a escribir de otra forma, es decir, brinda diversas rutas de acceso, en donde no se encarga de delimitar sino que se encarga de hacer un acercamiento más efectivo a la escritura a través de la creación. Para poder hacer la propuesta "El juego en la creación de cuentos en grado cuarto" se tuvo que recurrir a teóricos que se hubieran centrado en reconocer la escritura como parte de un constructo creativo, partiendo como ya se mencionó de Rodari (1983), continuando con Jean Michele Adam (1992), para comprender el significado de secuencia narrativa y teniendo en cuenta los aportes de Betuel Bonilla (2016).

Los nombrados anteriormente se han encargado de indagar sobre el cuento y para esto referencian a los autores más destacados en la literatura que pueden dar cuenta de este género, que es relevante cuando menciona a Juan Gabriel Vásquez porque este escritor colombiano destaca que: " El cuento es un género íntimo, sobre emociones concentradas, con pocos personajes, que suele prescindir del gran marco social, y por lo tanto necesita un zoom muy potente y a veces un microscopio" (2016: 222), el cuento debe ser una construcción tan bien hecha que pueda atraer al público, que el lector sienta ganas de saber que ocurrirá páginas más adelante, ese es el gran poder que tiene el cuento, que logra involucrar tanto al que escribe como al que lee.

De acuerdo con lo anterior se puede afirmar que el cuento es un género con gran relevancia pero que se destruye en el aula, se corrompe en su tránsito en la escuela, en donde es distorsionado y se desvirtúa la funcionalidad que 
tiene para fortalecer esta habilidad escrita. Se desconoce el trabajo que han realizado los diferentes teóricos y pedagogos respecto a la construcción de cuentos, los cuales permiten crear mundos posibles y jugar con la imaginación de los niños.

Por esta razón, es que se menciona que si los profesores de lengua y de otras áreas se dieran cuenta del gran potencial que tienen los estudiantes cuando escriben cuentos, no se destruiría su capacidad creadora, haciendo que busquen categorías que no contribuyen al proceso escritural. En tanto son vistas de forma rígida y memorística, esto es totalmente diferente a lo que se busca con el proyecto en donde se quiere que el sujeto tenga en cuenta elementos como son la coherencia y la cohesión, que hacen que un cuento logre impactar al interior de una población determinada y esto es lo que sucede con los estudiantes de grado cuarto que al proponerles crear un cuento o leer uno, se remiten automáticamente a los que les han repetido durante años, llegando a desconocer que el bagaje literario es muy amplio y por esta razón el interés de la propuesta es aprender a escribir y conocer a la vez diferente literatura que despierte en el estudiante las ganas de generar historias asombrosas como las que se llevan al aula.

Para esto se hizo la selección de varios cuentos que no son conocidos por ellos, como por ejemplo los cuentos de Anthony Brown, los cuales les permiten una mayor y mejor aproximación a la literatura infantil y a tener más ideas para construir sus propias historias. Se seleccionaron estos cuentos debido a que hizo que los niños se sintieran fascinados por aquello que iban a encontrar. Algunas de sus narraciones parten de situaciones cotidianas que se aproximan a la vida de los estudiantes, como el caso del cuento "Willy el tímido" en el que se narra acerca de un gorila que quería dejar de ser debilucho y para esto tuvo que hacer infinidad de actividades para demostrarle a los demás que podía ser más grande, llegando a convertirse en un héroe.

De esta manera es que se empiezan a brindar ideas innovadoras sobre cómo crear cuentos y se hace un distanciamiento de la lectura de cuentos clásicos como "Caperucita roja" historia conocida por la gran mayoría y que en ocasiones aburre a los niños, en tanto que son narraciones que no despiertan expectativa o interés porque ellos ya las conocen, distintas a las de Anthony Brown porque permiten el asombro y pensar que de situaciones sencillas se pueden crear narraciones interesantes y que se identifican con muchas de ellas. Por esta razón el cuento más que ser un tipo de texto que se va a 
construir, se pretende potenciar la escritura a través de estos cuentos que hacen una invitación a crear e imaginar a partir de momentos comunes algo inimaginable.

Posteriormente, una de las grandes problemáticas que se tiene en el aula es que es poca la literatura infantil que llega a manos de los niños porque algunos maestros no ven la necesidad de hacerlo porque están más interesados en que haga a la perfección el trazo de la letra a o que repita veinte veces la palabra hacer con h y con c. Por esta razón es importante que el bagaje que se les brinda a los estudiantes con lecturas como la de Isol permita que se les muestre un mundo diferente. Esto hace que la creatividad se pueda desbordar y que sus escritos puedan ser iguales o mejores a los leídos en el aula, lo que indica que la funcionalidad del cuento en este proyecto busca sensibilizar a ese escrito novato y brindarle herramientas nuevas.

Por otra parte, se hace énfasis en estos cuentos poco conocidos para ampliar el campo literario de los niños, así que no es solo hablar del cuento y su estructura, sino ver cómo los distintos escritores han hecho cuentos muy creativos que hacen que se tenga que leer una y otra vez para encontrar infinidad de detalles. Se hace la invitación para que el estudiante pueda iniciar su cuento de forma diferente a la enseñada, proponiéndole que construya paso a paso su cuento, teniendo en cuenta cada parte de su estructura y que escribir requiere un proceso cuidadoso y eliminar esa concepción que tienen algunos niños que escribir es aburrido porque se ha enseñado que escribir es llenar todas las hojas del cuaderno con palabras que no comprenden o peor aún con escritos que son copiados de otros textos cuando los mismo estudiantes podrían elaborarlos.

De esta forma es que se recurre a nuevas historias, a medida que se aprende acerca del cuento también se conoce literatura infantil en donde el beneficiado es el estudiante y es gracias a cuentos como los de Anthony Browne y Gianni Rodari que se ha logrado llevar a cabo este proyecto. En donde el estudiante percibe que hay algo innovador y que se parte desde otros cuentos y no desde los cuentos clásicos que en realidad poco aportan porque se ha agotado tanto la historia que causa distanciamiento o en muchas ocasiones es un recital de memoria acerca de la historia, generando reducción de estas historias que tenían potencial como lo hizo Triunfo Arciniegas al crear otra versión de "Caperucita Roja" cuento en el que Caperucita tenía una actiud de desprecio del lobo, quien comprende y entiende la escritura desde una perspectiva que 
aporta a eso que se llama escritura proceso, que es una escritura con sentido, planeada y que se reelabora una y otra vez, en la que tiene importancia lo que escribe el niño y a medida que se escribe se juega con las palabras con la imaginación tanto del niño como de los otros.

\section{El taller pedagógico como estrategia para fortalecer la escritura de cuentos.}

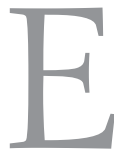

n cuanto a la forma de acercarse a esa experiencia de la escritura que se construye paso a paso, se hace uso del taller pedagógico para poder fortalecer los distintos aspectos escriturales de los estudiantes, en tanto se ha pensado que el taller es el medio que permite ir avanzando progresivamente y abarcar distintos componentes de la escritura, entendiendo el taller desde los postulados de Ander-Egg (1999) en el que se señala que el rol tanto del docente como el del profesor cambian, porque entre los dos se construye el conocimiento, en este caso se reconstruye aquello que se entiende por escritura.

De esta forma se busca que el sujeto logre ir perfeccionando su escritura, en tanto va recopilando y teniendo en cuenta todo lo que aprende en los talleres, pero más que centrarse en ubicar o utilizar aspectos formales, es que el individuo entienda que la escritura requiere de un proceso para que esta sea exitosa, es decir, que escribir es realizar un andamiaje que se hace de forma gradual.

Por tanto, el taller pedagógico permite hacer el seguimiento a lo que el estudiante crea y se complejiza su saber en tanto recuerda lo visto en cada uno de estos y mejora la estructura de su narración tanto a nivel semántico como sintáctico, haciendo que el estudiante aprende más cuando él se encuentra involucrado en la creación de su cuento, cuando es la persona que escribe lo que inventa y no mediado por consignas sencillas que no implican mayor esfuerzo.

Pasando a abordar la metodología que se utilizó para reconstruir la escritura, es la siguiente: fase número uno, en esta etapa se hizo una sensibilización, en la que el estudiante podía leer cuentos distintos y que lo atraparan como ese lector curioso y ávido de conocimiento, mostrado así que la literatura infantil es amplia, se inició con la escritura de un cuento de terror que permitiera desentrañar una primera escritura y observar cómo era la elaboración de cuentos y qué elementos eran empleados. En el segundo taller se apostó por 
el título de los cuentos, en el que se empieza a dimensionar la escritura de cuentos desde los detalles que aparentemente son sencillos. En este taller los estudiantes lograron aprender que más que iniciar a escribir un cuento de forma intrépida, se debe concebir que el título es el que atrae al lector y le brinda sentido al texto.

Continuando se desarrollaron talleres en los que se involucraba el uso de los conectores para enlazar las ideas, de la creación de párrafos para que generar una estructura, las partes del cuento (identificación consciente) y demás elementos que se fuero aprendiendo gracias al juego constante que se hacía con el lenguaje, en el que no se requería dar la definición de una determinada categoría, ya que lo importante es que el estudiante use distintos elementos en la práctica y no desde una definición aislada y que solo memoriza.

Es importante destacar que el proyecto se interesó en hacer el vínculo constante con la imaginación, el juego y las sensaciones- percepciones. De modo que los demás talleres se desarrollaron teniendo en cuenta las percepciones, en donde se invitaba al niño a contar cómo era su lugar favorito, si se componía de sabores, de olores y para esto se abordó el cuento "El edificio de helado" de Gianni Rodari, en el que un espacio tiene sabores exquisitos.

\section{A manera de conclusión}

través del proyecto que se gestó al interior de la Maestría en Pedagogía
de la Lengua Materna, se logró pensar y reflexionar la escritura desde
una perspectiva diferente, en donde el individuo tiene participación y es el gestor de sus cuentos, mostrándole de diversas formas que escribir no recae en la preocupación por identificar aspectos puntuales, sino que a medida que avanza puede ir incorporando elementos de nuestra lengua, pero que lo hace de forma progresiva, lo que afirma que primero debe hacerse un acercamiento al cuento de manera tal que el estudiante se sienta atraído por las historias que le cuentan o leen, para después dar paso a la escritura de historias más innovadoras que las leídas.

Al hablar de escritura proceso se hace referencia a esa escritura que busca que el autor pueda desentrañar lo que piensa y que lo haga de la forma como considera, a partir de ahí es que se empieza a mejorar su texto y que cada vez que lo lea vaya adquiriendo elementos de juicio para reconocer qué le hace falta a su texto o qué le sobra, pero este trabajo se hace más gratificante 
cuando se parte del cuento porque los niños se entusiasman cuando se cuenta algo asombroso o mágico y genera un acercamiento agradable para ellos, evidenciando gusto por la escritura y su interés por nutrir sus historias.

Finalmente, se hace alusión a los talleres pedagógicos porque gracias a esta estrategia se les va mostrando a los estudiantes diversos elementos de los cuentos y el empleo de estos en una historia, que tuvieron como fin último renovar y construir lo que es escribir en el aula, haciendo que los niños descubrieran por si solos que escribir tiene implicaciones sociales y que se comparte con los demás.

\section{REFERENCIAS BIBLIOGRÁFICAS}

Álvarez \& Cruz (2008). La escritura, lenguaje vivo en el aula: una experiencia de autoformación docente en didáctica. Enunciación 13(1), 1-8

Bernardin, J. (2014). Distancia y malentendidos frente a la escritura. Enunciación, 19(2), 366-376.

Cassany, Daniel (1995). La cocina de la escritura. Barcelona.

Cassany, Daniel (1999). Construir la escritura. Paidós. Barcelona.

Céspedes, Sandra (2014). Prácticas de lectura y escritura en contextos de educación rural. Maestría en Educación. Facultad de Educación. Universidad de Antioquia.

Ferreiro, Emilia (1998). La construcción de la escritura en el niño.

Ferreiro, Emilia (2001). Pasado y presente de los verbos leer y escribir. Fondo de Cultura Económica. México.

Nigro, Patricia (2006). Leer y escribir en la universidad: propuestas de articulación con la escuela media. En Dialnet.

Scardamalia \& Bereiter (1992). Dos modelos explicativos de los procesos de composición escrita. En la revista: Infancia y Aprendizaje. 

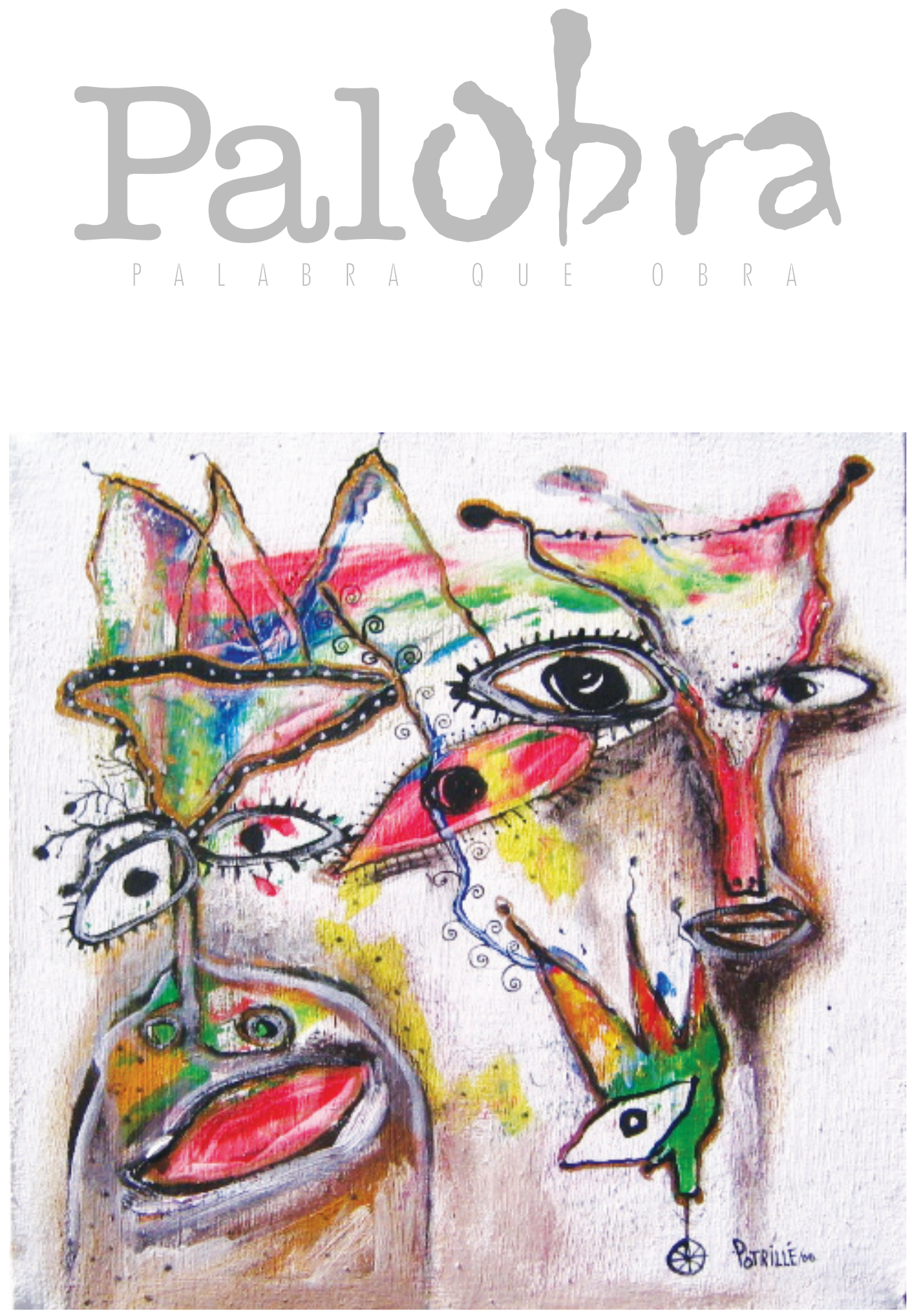

Obra pictórica de la serie "Rostros" de Eduardo Potrillé 